\title{
A generalized asymmetry model for square contingency tables with ordered categories
}

\author{
Kiyotaka Iki \\ Department of Information Sciences, Tokyo University of Science, \\ Noda City, Chiba, 278-8510, Japan \\ iki@is.noda.tus.ac.jp \\ Takahiro Suto \\ Department of Information Sciences, Tokyo University of Science, \\ Noda City, Chiba, 278-8510, Japan \\ tkhr.suto@gmail.com \\ Sadao Tomizawa \\ Department of Information Sciences, Tokyo University of Science, \\ Noda City, Chiba, 278-8510, Japan \\ tomizawa@is.noda.tus.ac.jp
}

Received 17 September 2015

Accepted 1 December 2015

\begin{abstract}
For square contingency tables with ordered categories, the present paper proposes an asymmetry model with $m$-additional parameters, which indicates (1) the generalized marginal homogeneity and (2) the structure of quasi-symmetry for cumulative probabilities. The proposed model includes a modified palindromic symmetry model by Iki, Oda and Tomizawa [7]. Also the present paper gives the decomposition of the symmetry model using the proposed model. Examples are given.

Keywords: Cumulative probability; Decomposition; Ordered category; Palindromic symmetry; Square contingency tables.
\end{abstract}

2010 Mathematics Subject Classification: 62H17

\section{Introduction}

Consider the square contingency tables with same row and column classifications. For example, consider the data in Table 1. The data in Table 1, taken from Goodman [5], are constructed from occupational status of 2391 farther-son pairs in Denmark. The row is the father's status category and column is the son's status category. The categories are ordered from (1) to (5) (high to low). In Table 1, many observations concentrate on the main diagonal cells. Therefore for these data, the model of independence does not hold. Namely, the father's status is strongly associated with the son's status. Instead, we are interested in whether or not a father's status is symmetric to his son's status. 
Table 1. Occupational status for Danish father-son pairs; from Goodman [5]. (Upper and lower parenthesized values are the maximum likelihood estimates of expected frequencies under the PS(3) and AS(3) models, respectively.)

\begin{tabular}{lcccccc}
\hline Father's & \multicolumn{5}{c}{ Son's status } & Total \\
\cline { 2 - 6 } status & $(1)$ & $(2)$ & $(3)$ & $(4)$ & $(5)$ & 57 \\
\hline$(1)$ & 18 & 17 & 16 & 4 & 2 & \\
& $(18.00)$ & $(18.48)$ & $(14.60)$ & $(4.41)$ & $(3.57)$ & \\
& $(18.00)$ & $(18.21)$ & $(14.07)$ & $(4.09)$ & $(4.05)$ & \\
$(2)$ & 24 & 105 & 109 & 59 & 21 & 318 \\
& $(22.75)$ & $(105.00)$ & $(105.59)$ & $(59.24)$ & $(17.29)$ & \\
& $(22.98)$ & $(105.00)$ & $(104.83)$ & $(56.46)$ & $(18.62)$ & \\
$(3)$ & 23 & 84 & 289 & 217 & 95 & 708 \\
& $(24.69)$ & $(87.14)$ & $(289.00)$ & $(220.85)$ & $(98.02)$ & \\
& $(25.44)$ & $(87.79)$ & $(289.00)$ & $(221.45)$ & $(95.50)$ & \\
$(4)$ & & & & & & \\
& $(7.44)$ & $(48.56)$ & $(171.11)$ & $(348.00)$ & $(195.96)$ & \\
& $(7.90)$ & $(51.37)$ & $(170.60)$ & $(348.00)$ & $(196.33)$ & \\
& & & & & & \\
$(5)$ & 6 & 8 & 69 & 201 & 246 & 530 \\
& $(5.01)$ & $(10.98)$ & $(65.98)$ & $(203.34)$ & $(246.00)$ & \\
& $(4.20)$ & $(9.70)$ & $(68.51)$ & $(202.91)$ & $(246.00)$ & \\
\hline Total & 79 & 263 & 658 & 829 & 562 & 2391 \\
\hline
\end{tabular}

Note: Status (1) is High professionals, (2) White-collar employees of higher education, (3) White-collar employees of less high education, (4) Upper working class, and (5) Unskilled workers.

For an $r \times r$ square contingency table with the same row and column ordinal classifications, let $p_{i j}$ denote the probability that an observation will fall in the $i$ th row and $j$ th column of the table $(i=1, \ldots, r ; j=1, \ldots, r)$. The symmetry ( $\mathrm{S})$ model is defined by

$$
p_{i j}=p_{j i} \quad(i<j)
$$

see Bowker [3], Bishop, Fienberg and Holland [2, p. 282] and Agresti [1, Chap. 11]. For the data in Table 1, the $\mathrm{S}$ model indicates that the probability that a father's status is $i$ and his son's status is $j$, is equal to the probability that the father's status is $j$ and his son's status is $i$. Namely, this model describes a structure of symmetry of the probabilities $\left\{p_{i j}\right\}$ with respect to the main diagonal of the table. Let $X_{1}$ and $X_{2}$ denote the row and column variables, respectively, and let

$$
G_{i j}=\sum_{s=1}^{i} \sum_{t=j}^{r} p_{s t}=\mathrm{P}\left(X_{1} \leq i, X_{2} \geq j\right) \quad(i<j),
$$

and

$$
G_{j i}=\sum_{s=j}^{r} \sum_{t=1}^{i} p_{s t}=\mathrm{P}\left(X_{1} \geq j, X_{2} \leq i\right) \quad(i<j) .
$$

The $\mathrm{S}$ model may be expressed as

$$
G_{i j}=G_{j i} \quad(i<j)
$$


For the data in Table 1, this indicates that the cumulative probability that a father's status is $i$ or below and his son's status is $j$ or above, is equal to the cumulative probability that the father's status is $j$ or above and his son's status is $i$ or below. The marginal homogeneity (MH) model (Stuart [12]) is defined by

$$
p_{i \cdot}=p_{\cdot i} \quad(i=1, \ldots, r),
$$

where $p_{i \cdot}=\sum_{t=1}^{r} p_{i t}$ and $p_{\cdot i}=\sum_{s=1}^{r} p_{s i}$. The MH model may be expressed as

$$
G_{i, i+1}=G_{i+1, i} \quad(i=1, \ldots, r-1) .
$$

Tahata and Tomizawa [13] considered the $m$-additional parameters marginal homogeneity $(\mathrm{MH}(m))$ model which is a generalization of the MH model. For a given $m(m=1, \ldots, r-1)$, the $\mathrm{MH}(m)$ model is defined by

$$
G_{i, i+1}=\Delta_{i}^{(m)} G_{i+1, i} \quad(i=1, \ldots, r-1)
$$

where

$$
\Delta_{i}^{(m)}=\prod_{k=0}^{m-1} \Delta_{k}^{i^{k}}
$$

A special case of the $\mathrm{MH}(m)$ model obtained by putting $\left\{\Delta_{k}=1\right\}$ is the MH model. When $m=1,2$ and $r-1$, the $\mathrm{MH}(m)$ models are the extended marginal homogeneity model in Tomizawa [16], the generalized marginal homogeneity model in Tomizawa [17] and saturated model, respectively.

Caussinus [4] proposed the quasi-symmetry (QS) model for cell probabilities, defined by

$$
p_{i j}=\alpha_{i} \beta_{j} \psi_{i j} \quad(i=1, \ldots, r ; j=1, \ldots, r),
$$

where $\psi_{i j}=\psi_{j i}$ (see also Goodman [6]; Kateri and Agresti [8]). Denote the odds ratio for rows $i$ and $s(>i)$, and columns $j$ and $t(>j)$ by $\theta_{i s ; j t}$. Thus $\theta_{i s, j t}=\left(p_{i j} p_{s t}\right) /\left(p_{i t} p_{s j}\right)$. For the data in Table 1 , the $\theta_{i s ; j t}\left(=\left(p_{i j} / p_{s j}\right) /\left(p_{i t} / p_{s t}\right)\right)$ indicates that the ratio of the odds that the father's status is $i$ instead of $s$ when the son's status is $j$ to the odds that the father's status is $i$ instead of $s$ when the son's status is $t$. Using odds ratios, the QS model is also expressed as

$$
\theta_{i s ; j t}=\theta_{j t ; i s} \quad(i<s ; j<t) .
$$

Therefore this model has characterization in terms of symmetry of odds ratios (though the $\mathrm{S}$ model has characterization in terms of symmetry of cell probabilities). The QS model may be expressed as

$$
p_{i j}=\xi_{i} \phi_{i j} \quad(i=1, \ldots, r ; j=1, \ldots, r),
$$

where $\phi_{i j}=\phi_{j i}$. Caussinus [4] also gave the theorem that the S model holds if and only if the QS and MH models hold. Miyamoto, Ohtsuka and Tomizawa [10] proposed the cumulative quasi-symmetry (CQS) model for cumulative probabilities $\left\{G_{i j}\right\}$, defined by

$$
G_{i j}=\gamma_{i} \Psi_{i j} \quad(i \neq j), \quad p_{i i}=\Psi_{i i},
$$

where $\Psi_{i j}=\Psi_{j i}$. This model may be expressed as

$$
\frac{G_{i j}}{G_{j i}}=\frac{\gamma_{i}}{\gamma_{j}} \quad(i<j)
$$

see also Tahata and Tomizawa [14]. 
McCullagh [9] considered the palindromic symmetry (PS) model, defined by

$$
G_{i j}=\left\{\begin{array}{c}
\Delta \alpha_{i} \Psi_{i j}(i<j), \\
\alpha_{i-1} \Psi_{i j}(i>j),
\end{array} \quad p_{i i}=\Psi_{i i}\right.
$$

where $\Psi_{i j}=\Psi_{j i}$ and $\alpha_{1}=1$ without loss of generality. A special case of the PS model obtained by putting $\Delta=1$ and $\left\{\alpha_{i}=1\right\}$ is the S model. Note that the PS model with $\Delta$ replaced by $\Delta_{i}$ is the generalized palindromic symmetry model (McCullagh [9]). The PS model is also expressed as

$$
\frac{G_{i j}}{G_{j i}}=\Delta \frac{\alpha_{i}}{\alpha_{j-1}} \quad(i<j)
$$

Saigusa, Tahata and Tomizawa [11] considered the $m$-additional parameters palindromic symmetry (PS $(m))$ model. For a given $m(m=1, \ldots, r-1)$, the PS $(m)$ model is defined by

$$
\frac{G_{i j}}{G_{j i}}=\Delta_{i}^{(m)} \frac{\alpha_{i}}{\alpha_{j-1}} \quad(i<j)
$$

where

$$
\Delta_{i}^{(m)}=\prod_{k=0}^{m-1} \Delta_{k}^{i^{k}}
$$

When $m=1$ (with $\Delta_{i}^{(1)}=\Delta_{0}$ ), the PS(1) model is identical to the PS model, and when $m=r-1$, the PS $(r-1)$ model is the generalized palindromic symmetry model. Iki, Oda and Tomizawa [7] considered the modified palindromic symmetry (MPS) model. The MPS model is defined by

$$
G_{i j}=\left\{\begin{array}{l}
\beta_{i} \Psi_{i j}(i<j ; j \neq i+1), \\
\Gamma \beta_{i} \Psi_{i j}(i<r ; j=i+1), \quad p_{i i}=\Psi_{i i}, \\
\beta_{i-1} \Psi_{i j}(i>j)
\end{array}\right.
$$

where $\Psi_{i j}=\Psi_{j i}$ and $\beta_{1}=1$ without loss of generality. A special case of this model obtained by putting $\Gamma=1$ and $\left\{\beta_{i}=1\right\}$ is the $\mathrm{S}$ model. The MPS model is also expressed as

$$
\frac{G_{i, i+1}}{G_{i+1, i}}=\Gamma \quad(i=1, \ldots, r-1),
$$

and

$$
\frac{G_{i j}}{G_{j i}}=\frac{\beta_{i}}{\beta_{j-1}} \quad(i<j ; j \neq i+1) .
$$

The PS $(m)$ model is the PS model with the $m$-additional parameters $\Delta_{i}^{(m)}$ instead of one parameter $\Delta$. So, we are interested in a model which is the MPS model with the $m$-additional parameters instead of one parameter $\Gamma$.

In the present paper, Section 2 proposes a new model which is the MPS model with the $m$ additional parameters. Section 3 gives the decomposition of the $\mathrm{S}$ model using the proposed model. Section 4 describes the goodness-of-fit test and Section 5 gives examples. Section 6 provides some concluding remarks. 


\section{An asymmetry model with $m$-additional parameters}

Consider a model defined by, for a given $m(m=1, \ldots, r-1)$,

$$
G_{i j}=\left\{\begin{array}{cl}
\beta_{i} \Psi_{i j} & (i<j ; j \neq i+1), \\
\Gamma_{i}^{(m)} \beta_{i} \Psi_{i j} & (i<r ; j=i+1), \quad p_{i i}=\Psi_{i i}, \\
\beta_{i-1} \Psi_{i j} & (i>j),
\end{array}\right.
$$

where $\Psi_{i j}=\Psi_{j i}, \beta_{1}=1$ without loss of generality and

$$
\Gamma_{i}^{(m)}=\prod_{k=0}^{m-1} \Gamma_{k}^{i^{k}}
$$

We shall refer to this model as the $m$-additional parameters asymmetry (AS $(m))$ model. Especially, the AS(1) model is identical to the MPS model. The AS $(m)$ model is also expressed as

$$
\frac{G_{i, i+1}}{G_{i+1, i}}=\Gamma_{i}^{(m)} \quad(i=1, \ldots, r-1),
$$

and

$$
\frac{G_{i j}}{G_{j i}}=\frac{\beta_{i}}{\beta_{j-1}} \quad(i<j ; j \neq i+1)
$$

From the equation (2.1), the parameter $\Gamma_{i}^{(m)}$ indicates that the ratio of the cumulative probability that an observation will fall in row category $i$ or below and column category $i+1$ or above (i.e., $G_{i, i+1}$ ) to the cumulative probability that the observation falls in row category $i+1$ or above and column category $i$ or below (i.e., $\left.G_{i+1, i}\right)$. In addition, the equation (2.1) is identical to the $\mathrm{MH}(m)$ model. Namely, the log-odds of cumulative probabilities are expressed as the polynomial function of category indicator $i(i=1, \ldots, r-1)$, i.e.,

$$
\log \left(\frac{G_{i, i+1}}{G_{i+1, i}}\right)=\log \Gamma_{0}+i \log \Gamma_{1}+\cdots+i^{m-1} \log \Gamma_{m-1}
$$

The equation (2.2) states that the cumulative probability that an observation will fall in row category $i$ or below and column category $j(i<j ; j \neq i+1)$ or above, is $\beta_{i} / \beta_{j-1}$ times higher than the cumulative probability that the observation falls in row category $j$ or above and column category $i$ or below. From $\beta_{1}=1$, the parameters $\beta_{j-1}$ indicates the ratio of $G_{j 1}$ to $G_{1 j}(j=3, \ldots, r)$. The $\mathrm{AS}(m)$ model is expressed as the equations (2.1) and (2.2), the interpretation of the parameters $\left\{\Psi_{i j}\right\}$ is not essential.

Define the odds ratio based on $\left\{G_{i j}\right\}, i \neq j$, by $\Theta_{i s ; j t}=\left(G_{i j} G_{s t}\right) /\left(G_{i t} G_{s j}\right)$ for $1 \leq i<s<j<$ $t \leq r$. For the data in Table 1, the $\Theta_{i s ; j t}\left(=\left(G_{i j} / G_{s j}\right) /\left(G_{i t} / G_{s t}\right)\right)$ indicates that the ratio of the odds that the father's status is $i$ or below instead of $s$ or below when the son's status is $j$ or above to the odds that the father's status is $i$ or below instead of $s$ or below when the son's status is $t$ or above. 
The PS $(m)$ model implies

$$
\Theta_{i s ; j t}=\Theta_{j t ; i s} \quad(1 \leq i<s<j<t \leq r) .
$$

The AS $(m)$ model also implies

$$
\Theta_{i s ; j t}=\Theta_{j t ; i s} \quad(1 \leq i<s<j<t \leq r ; j \neq s+1),
$$

and

$$
\Gamma_{s}^{(m)} \Theta_{i s ; j t}=\Theta_{j t ; i s} \quad(1 \leq i<s<j<t \leq r ; j=s+1) .
$$

Therefore, PS $(m)$ model implies the symmetry of odds ratios based on $\left\{G_{i j}\right\}, i \neq j$; however, the AS $(m)$ model implies the symmetry of odds ratios with the asymmetry partially. Note that the PS $(m)$ and $\mathrm{AS}(m)$ models have the structure of constant of odds $\left\{G_{i, i+1} / G_{i+1, i}\right\}, i=1, \ldots, r-1$.

\section{Decomposition of symmetry model}

Tomizawa, Miyamoto and Ouchi [18] proposed the cumulative subsymmetry (CS) model, defined by

$$
G_{i, i+2}=G_{i+2, i} \quad(i=1, \ldots, r-2) .
$$

For a given positive integer $s$, we consider the sth moment equality $(\mathrm{ME}(s))$ model defined by

$$
\mathrm{E}\left(X_{1}^{s}\right)=\mathrm{E}\left(X_{2}^{s}\right),
$$

where

$$
\mathrm{E}\left(X_{1}^{s}\right)=\sum_{i=1}^{r} i^{s} p_{i .}, \quad \mathrm{E}\left(X_{2}^{s}\right)=\sum_{i=1}^{r} i^{s} p_{. i} .
$$

We obtain the decomposition of the $\mathrm{S}$ model as follows:

Theorem 3.1. For a given $m(m=1, \ldots, r-1)$, the $S$ model holds if and only if all the AS( $m), C S$ and $M E(s)(s=1, \ldots, m)$ models hold.

Proof. If the S model holds, then for a given $m(m=1, \ldots, r-1)$, the $\operatorname{AS}(m), \operatorname{CS}$ and $\operatorname{ME}(s)$ $(s=1, \ldots, m)$ models hold. Assuming that all the AS $(m), \mathrm{CS}$ and $\{\mathrm{ME}(s)\}$ models hold, then we shall show that the $\mathrm{S}$ model holds. From the AS $(m)$ and CS models, we see

$$
\frac{G_{i, i+2}}{G_{i+2, i}}=\frac{\beta_{i}}{\beta_{i+1}}=1 \quad(i=1, \ldots, r-2) .
$$

Since $\beta_{1}=1$, thus we see $\left\{\beta_{i}=1\right\}$. From the AS $(m)$ model holds, we see

$$
\frac{G_{i, i+1}}{G_{i+1, i}}=\Gamma_{i}^{(m)} \quad(i=1, \ldots, r-1) .
$$

A structure of $\left\{G_{i, i+1} / G_{i+1, i}\right\}$ in the $\operatorname{AS}(m)$ model is identical to that of the $\mathrm{MH}(m)$ model. Tahata and Tomizawa [13] showed that, for a given $m(m=1, \ldots, r-1)$, the MH model holds if and only if all the $\operatorname{MH}(m)$ and $\operatorname{ME}(s)(s=1, \ldots, m)$ models hold. Therefore, the model which satisfies all 
constraints of $\left\{G_{i, i+1} / G_{i+1, i}\right\}$ in the $\operatorname{AS}(m)$ model and $\operatorname{ME}(s)(s=1, \ldots, m)$ model is identical to the MH model. Namely,

$$
\frac{G_{i, i+1}}{G_{i+1, i}}=1 \quad(i=1, \ldots, r-1) .
$$

Thus, we obtain $\Gamma_{k}=1$ for $k=0,1, \ldots, m-1$. Therefore, the $\mathrm{S}$ model holds. The proof is completed.

\section{Goodness-of-fit test}

Let $n_{i j}$ denote the observed frequency in the $i$ th row and $j$ th column of the $r \times r$ table $(i=$ $1, \ldots, r ; j=1, \ldots, r)$ with $n=\sum \sum n_{i j}$. Assume that a multinomial distribution applies to the $r \times r$ table. The maximum likelihood estimates of expected frequencies under models could be obtained by using the Newton-Raphson method in the log-likelihood equation. We can test each model for goodness-of-fit by the likelihood ratio chi-squared statistic (denoted by $G^{2}$ ) with the corresponding degrees of freedom. The test statistic $G^{2}$ is defined by

$$
G^{2}=2 \sum_{i=1}^{r} \sum_{j=1}^{r} n_{i j} \log \left(\frac{n_{i j}}{\widehat{m}_{i j}}\right),
$$

where $\widehat{m}_{i j}$ is the maximum likelihood estimate of expected frequency $m_{i j}$ under the model.

For nested models, the conditional test statistic would be the $G^{2}$ value for the model with fewer free parameters minus the $G^{2}$ value for the model with more free parameters, which has a chi-square distribution with degrees of freedom equal to the distance between numbers of parameters in the two models.

The number of degrees of freedom for the $\operatorname{AS}(m)$ model is $\frac{1}{2}\left(r^{2}-3 r+4\right)-m$ for $m=$ $1,2, \ldots, r-1$. This number is identical to that of the PS $(m)$ model.

\section{Examples}

Example 1. Consider the data in Table 1 again. Table 2 gives the values of likelihood ratio statistic $G^{2}$ for testing the goodness-of-fit of some models. The $\mathrm{S}$ model fits these data poorly. Also, each of the PS(1), PS(2), $\operatorname{AS}(1), \operatorname{AS}(2), \operatorname{CS}$ and $\operatorname{ME}(s), s=1,2,3,4$ models fits poorly, but each of the PS(3), PS(4), AS(3) and AS(4) models fits the data well.

According to the test based on the difference between the likelihood ratio chi-squared values $G^{2}$ for the AS(3) and AS(4) models at the 0.05 significance level, we obtain the AS(3) model is preferable to the AS(4) model. In the similar way, the PS(3) model is preferable to the PS(4) model.

Under the AS(3) model, the maximum likelihood estimates of parameters $\Gamma_{0}, \Gamma_{1}$ and $\Gamma_{2}$ are $\widehat{\Gamma}_{0}=0.299, \widehat{\Gamma}_{1}=2.614$ and $\widehat{\Gamma}_{2}=0.853$, respectively. The cumulative probability that the father's status is $i$ or below and the son's status is $i+1$ or above, is estimated to be $\widehat{\Gamma}_{0} \widehat{\Gamma}_{1}^{i} \widehat{\Gamma}_{2}^{i^{2}}$ times higher than the cumulative probability that the father's status is $i+1$ or above and the son's status is $i$ or below. When $i=1$, the cumulative probability that the father's status is '(1)' and his son's status is '(2)' to '(5)', is estimated to be $\widehat{\Gamma}_{0} \widehat{\Gamma}_{1} \widehat{\Gamma}_{2}=0.668$ times higher than the cumulative probability that the father's status is '(2)' to '(5)' and his son's status is '(1)'. Besides, the maximum likelihood estimates of $\left\{\beta_{i}\right\}$ are $\widehat{\beta}_{2}=1.690, \widehat{\beta}_{3}=1.486$ and $\widehat{\beta}_{4}=1.036$, with $\widehat{\beta}_{1}=1$. For $i<j$ with $j-i \geq 2$, the probability that the father's status is $i$ or below and the son's status is $j$ or above is estimated to be $\widehat{\beta}_{i} / \widehat{\beta}_{j-1}$ times higher than the probability that the father's status is $j$ or above and the son's status 
Table 2. Likelihood ratio chi-squared values $G^{2}$ for models applied to the data in Table 1 .

\begin{tabular}{lcc}
\hline Models & Degree of freedom & $G^{2}$ \\
\hline S & 10 & $24.80^{*}$ \\
$\mathrm{PS}(1)$ & 6 & $15.07^{*}$ \\
$\mathrm{PS}(2)$ & 5 & $14.42^{*}$ \\
$\mathrm{PS}(3)$ & 4 & 3.82 \\
$\mathrm{PS}(4)$ & 3 & 2.60 \\
$\mathrm{AS}(1)$ & 6 & $15.40^{*}$ \\
$\mathrm{AS}(2)$ & 5 & $14.35^{*}$ \\
$\mathrm{AS}(3)$ & 4 & 3.99 \\
$\mathrm{AS}(4)$ & 3 & 3.21 \\
$\mathrm{CS}$ & 3 & $12.07^{*}$ \\
$\mathrm{ME}(1)$ & 1 & $5.73^{*}$ \\
$\mathrm{ME}(2)$ & 1 & $6.86^{*}$ \\
$\mathrm{ME}(3)$ & 1 & $6.58^{*}$ \\
ME(4) & 1 & $5.80^{*}$ \\
${ }^{*}$ means significant at the 0.05 level.
\end{tabular}

Table 3. Values of $\left\{\widehat{G}_{i j} / \widehat{G}_{j i}\right\}, i \neq j$, under the $\operatorname{AS}(3)$ and PS(3) models applied to Table 1.

\begin{tabular}{cccccc}
\multicolumn{7}{c}{ (a) Under the $\mathrm{AS}(3)$ model } \\
\hline \multicolumn{7}{c}{} & $j=1$ & 2 & 3 & 4 & 5 \\
\hline$i=1$ & - & 0.668 & 0.592 & 0.673 & 0.965 \\
2 & - & - & 1.084 & 1.138 & 1.631 \\
3 & - & - & - & 1.281 & 1.434 \\
4 & - & - & - & - & 1.102 \\
5 & - & - & - & - & - \\
\hline
\end{tabular}

\begin{tabular}{cccccc}
\multicolumn{7}{l}{ (b) Under the PS(3) model } \\
\hline \multicolumn{7}{c}{} & $j=1$ & 2 & 3 & 4 & 5 \\
\hline$i=1$ & - & 0.686 & 0.608 & 0.641 & 0.712 \\
2 & - & - & 1.114 & 1.174 & 1.305 \\
3 & - & - & - & 1.305 & 1.450 \\
4 & - & - & - & - & 1.103 \\
5 & - & - & - & - & - \\
\hline
\end{tabular}

is $i$ or below. When $i=1$ and $j=3$, the cumulative probability that the father's status is '(1)' and his son's status is '(3)' to '(5)', is estimated to be $\widehat{\beta}_{1} / \widehat{\beta}_{2}=0.592$ times higher than the cumulative probability that the father's status is '(3)' to '(5)' and his son's status is '(1)'. Under the PS(3) model, the maximum likelihood estimates of parameters $\Delta_{0}, \Delta_{1}$ and $\Delta_{2}$ are $\widehat{\Delta}_{0}=0.305, \widehat{\Delta}_{1}=2.651$ and $\widehat{\Delta}_{2}=0.849$, respectively. Besides, the maximum likelihood estimates of $\left\{\alpha_{i}\right\}$ are $\widehat{\alpha}_{2}=1.127$, $\widehat{\alpha}_{3}=1.070$ and $\widehat{\alpha}_{4}=0.963$, with $\widehat{\alpha}_{1}=1$. From Table 3 , we see that the values of $\left\{G_{i j} / G_{j i}\right\}, i \neq j$, under the AS(3) model are close to those under the PS(3) model.

Furthermore, under the $\mathrm{AS}(3)$ model, the odds ratio $\theta_{34 ; 12}$ is estimated to be $\widehat{\Gamma}_{2}^{(3)}=1.084$ times greater than the odds ratio $\theta_{12 ; 34}$. Hence the ratio of the odds that the son's status is '(1)' instead of '(1)' or '(2)' when the father's status is '(3)' or '(4)' to the odds when the father's status is '(4)', is estimated to be $\widehat{\Gamma}_{2}^{(3)}=1.084$ times greater than the ratio of the odds that the father's status is '(1)' instead of '(1)' or '(2)' when the son's status is '(3)' or '(4)' to the odds when the son's status is 
'(4)'. Also, the odds ratio $\theta_{45 ; 23}$ is estimated to be $\widehat{\Gamma}_{3}^{(3)}=1.281$ times greater than the odds ratio $\theta_{23 ; 45}$, and interpreted similarly.

Example 2. The data in Table 4, taken from Stuart [12], are constructed from unaided distance vision of 7477 women aged 30-39 employed in Royal Ordnance factories in Britain from 1943 to 1946.

Table 4. Unaided distance vision of 7477 women aged 30-39 employed in Royal Ordnance factories in Britain from 1943 to 1946; from Stuart ([12]. (The upper, middle and lower parenthesized values are the maximum likelihood estimates of expected frequencies under the $\operatorname{AS}(1), \operatorname{AS}(2)$ and $\operatorname{AS}(3)$ models, respectively.)

\begin{tabular}{cccccc}
\hline Right eye & \multicolumn{4}{c}{ Left eye grade } & \\
\cline { 2 - 5 } grade & Best (1) & Second (2) & Third (3) & Worst (4) & Total \\
\hline Best (1) & 1520 & 266 & 124 & 66 & 1976 \\
& $(1520.00)$ & $(266.46)$ & $(129.43)$ & $(64.22)$ & \\
& $(1520.00)$ & $(266.55)$ & $(129.47)$ & $(64.21)$ & \\
& $(1520.00)$ & $(266.00)$ & $(129.49)$ & $(64.10)$ & \\
Second (2) & 234 & 1512 & 432 & 78 & 2256 \\
& $(233.52)$ & $(1512.00)$ & $(430.86)$ & $(82.71)$ & \\
& $(233.42)$ & $(1512.00)$ & $(430.84)$ & $(82.68)$ & \\
& $(234.00)$ & $(1512.00)$ & $(432.00)$ & $(82.62)$ & \\
Third (3) & 117 & & & & \\
& $(110.99)$ & $(363.15)$ & $(1772.00)$ & $(205.63)$ & \\
& $(110.94)$ & $(363.17)$ & $(1772.00)$ & $(205.55)$ & \\
& $(110.94)$ & $(362.00)$ & $(1772.00)$ & $(205.00)$ & \\
Worst (4) & 36 & & & & \\
& $(39.22)$ & $(76.47)$ & $(178.34)$ & $(492.00)$ & \\
& $(39.22)$ & $(76.51)$ & $(178.42)$ & $(492.00)$ & \\
& $(39.28)$ & $(76.58)$ & $(179.00)$ & $(492.00)$ & \\
\hline Total & 1907 & 2222 & 2507 & 841 & 7477 \\
\hline
\end{tabular}

Table 5 gives the values of likelihood ratio statistic $G^{2}$ for testing the goodness-of-fit of some models. The S, PS(2), PS(3) and $\operatorname{ME}(s), s=1,2,3$ models fit poorly, but each of the PS(1), $\operatorname{AS}(m)$, $m=1,2,3$ and CS models fits the data well. Using Theorem 3.1, it is inferred that, e.g., when $m=3$, the poor fit of $\mathrm{S}$ model is caused by the influence of the lack of structure of the $\mathrm{ME}(s), s=1,2,3$ models rather than those of the CS and AS(3) models.

From Table 5, we can see the likelihood ratio chi-squared values $G^{2}$ of $\operatorname{AS}(m), m=1,2,3$ models are almost the same. Under the AS(1) model, the maximum likelihood estimate of parameter $\Gamma_{0}$ is $\widehat{\Gamma}_{0}=1.199$. Under the AS(2) model, the maximum likelihood estimates of parameters $\Gamma_{0}$ and $\Gamma_{1}$ are $\widehat{\Gamma}_{0}=1.201$ and $\widehat{\Gamma}_{1}=0.999$, respectively. Besides, $\widehat{\Gamma}_{0} \widehat{\Gamma}_{1}=1.200, \widehat{\Gamma}_{0} \widehat{\Gamma}_{1}^{2}=1.199$ and $\widehat{\Gamma}_{0} \widehat{\Gamma}_{1}^{3}=$ 1.198. Under the AS(3) model, the maximum likelihood estimates of parameters $\Gamma_{0}, \Gamma_{1}$ and $\Gamma_{2}$ are $\widehat{\Gamma}_{0}=1.173, \widehat{\Gamma}_{1}=1.027$ and $\widehat{\Gamma}_{2}=0.993$, respectively. Besides, $\widehat{\Gamma}_{0} \widehat{\Gamma}_{1} \widehat{\Gamma}_{2}=1.196, \widehat{\Gamma}_{0} \widehat{\Gamma}_{1}^{2} \widehat{\Gamma}_{2}^{4}=1.203$ and $\widehat{\Gamma}_{0} \widehat{\Gamma}_{1}^{3} \widehat{\Gamma}_{2}^{9}=1.193$.

According to the test based on the difference between the likelihood ratio chi-squared values $G^{2}$ for $\mathrm{AS}(1)$ and $\mathrm{AS}(2)$ models (also for $\mathrm{AS}(2)$ and $\mathrm{AS}(3)$ models), the $\mathrm{AS}(1)$ model is preferable to 


\begin{tabular}{|c|c|c|}
\hline Models & Degree of freedom & $G^{2}$ \\
\hline S & 6 & $19.25 *$ \\
\hline $\mathrm{PS}(1)$ & 3 & 6.24 \\
\hline $\operatorname{PS}(2)$ & 2 & $6.24 *$ \\
\hline $\mathrm{PS}(3)$ & 1 & $6.18^{*}$ \\
\hline $\mathrm{AS}(1)$ & 3 & 1.55 \\
\hline $\mathrm{AS}(2)$ & 2 & 1.55 \\
\hline $\operatorname{AS}(3)$ & 1 & 1.54 \\
\hline $\mathrm{CS}$ & 2 & 5.00 \\
\hline $\operatorname{ME}(1)$ & 1 & $11.98 *$ \\
\hline $\operatorname{ME}(2)$ & 1 & $11.30 *$ \\
\hline $\operatorname{ME}(3)$ & 1 & $10.00 *$ \\
\hline
\end{tabular}

the AS(2) and AS(3) models. Under the AS(1) model, for example, the cumulative probability that a woman's right eye grade is Best (1) and her left eye grade is Second (2) to Worst (4), is estimated to be $\widehat{\Gamma}_{0}=1.199$ times higher than the cumulative probability that the woman's right eye grade is Second (2) to Worst (4) and her left eye grade is Best (1).

\section{Concluding remarks}

We have proposed the AS $(m)$ model for $m=1,2, \ldots, r-1$. Especially when $m=1$, the AS(1) model is identical to the MPS model. Compare the $\operatorname{AS}(m)$ and the PS $(m)$ models. Both of the AS $(m)$ and PS $(m)$ models have the structure of $\mathrm{MH}(m)$ model. The AS $(m)$ model has the structure of CQS model for $\left\{G_{i j}\right\}$ with $|j-i| \geq 2$, although the PS $(m)$ model does not have the such structure. As seen in Example 1, when we analyze the data using the MPS model (i.e., AS(1) model), the structure of asymmetry in MPS model with $G_{i, i+1} / G_{i+1, i}=\Gamma_{0}$ may be strong restriction. The addition of parameters in the AS $(m)$ model relative to the $\mathrm{S}$ model improves the fit. However, the more we use the parameters in the $\operatorname{AS}(m)$ model, the more complicated the interpretation for the $\operatorname{AS}(m)$ model becomes. The AS $(m)$ model implies the structure of symmetry of odds ratios $\left\{\theta_{i j ; s t}\right\}$ based on $\left\{G_{i j}\right\}$, $i \neq j$, with the structure of asymmetry partially (being $\Gamma_{j}^{(m)} \theta_{i j ; s t}=\theta_{s t ; i j}$ for $1 \leq i<j<s<t \leq r$ and $s=j+1)$. However, PS $(m)$ model has the structure of symmetry of odds ratio. Therefore the AS $(m)$ model would be useful for seeing the mixed structure of symmetry plus asymmetry of odds ratios for cumulative probabilities $\left\{G_{i j}\right\}, i \neq j$.

The decomposition of the $\mathrm{S}$ model into the $\mathrm{AS}(m), \mathrm{CS}$ and $\operatorname{ME}(s)(s=1,2, \ldots, m)$ models, given by Theorem 3.1, would be useful for seeing the reason for its poor fit when the $S$ model fits the data poorly (see Example 2).

\section{Acknowledgments}

The authors would like to thank the referees for their helpful comments.

\section{References}

[1] A. Agresti, Analysis of Ordinal Categorical Data, Third edition. (Wiley, New Jersey, 2013).

[2] Y.M.M. Bishop, S.E. Fienberg and P.W. Holland, Discrete Multivariate Analysis: Theory and Practice. (The MIT Press, Cambridge, 1975). 
[3] A.H. Bowker, A test for symmetry in contingency tables, Journal of the American Statistical Association. 43 (1948) 572-574.

[4] H. Caussinus, Contribution à l'analyse statistique des tableaux de corrélation, Annales de la Faculté des Sciences de l'Université de Toulouse. 29 (1965) 77-182.

[5] L.A. Goodman, Association models and the bivariate normal for contingency tables with ordered categories, Biometrika. 68 (1981) 347-355.

[6] L.A. Goodman, The analysis of cross-classified data having ordered and/or unordered categories: Association models, correlation models, and asymmetry models for contingency tables with or without missing entries, Annals of Statistics. 13 (1985) 10-69.

[7] K. Iki, T. Oda and S. Tomizawa, A modified palindromic symmetry model for square contingency tables with ordered categories, Journal of Statistics Applications and Probability. 3 (2014) 109-115.

[8] M. Kateri and A. Agresti, A class of ordinal quasi-symmetry models for square contingency tables, Statistics and Probability Letters. 77 (2007) 598-603.

[9] P. McCullagh, A class of parametric models for the analysis of square contingency tables with ordered categories, Biometrika. 65 (1978) 413-418.

[10] N. Miyamoto, W. Ohtsuka and S. Tomizawa, Linear diagonals-parameter symmetry and quasisymmetry models for cumulative probabilities in square contingency tables with ordered categories, Biometrical Journal. 46 (2004) 664-674.

[11] Y. Saigusa, K. Tahata and S. Tomizawa, An extended asymmetry model for square contingency tables with ordered categories, Model Assisted Statistics and Applications: An International Journal. 9 (2014) 151-157.

[12] A. Stuart, A test for homogeneity of the marginal distributions in a two-way classification, Biometrika. 42 (1955) 412-416.

[13] K. Tahata and S. Tomizawa, Generalized marginal homogeneity model and its relation to marginal equimoments for square contingency tables with ordered categories, Advances in Data Analysis and Classification. 2 (2008) 295-311.

[14] K. Tahata and S. Tomizawa, Symmetry and asymmetry models and decompositions of models for contingency tables, SUT Journal of Mathematics. 50 (2015) 131-165.

[15] K. Tahata, K. Yamamoto and S. Tomizawa, Decomposition of symmetry using palindromic symmetry model in a two-way classification, Journal of Statistics Applications and Probability. 1 (2012) 175-178.

[16] S. Tomizawa, Three kinds of decompositions for the conditional symmetry model in a square contingency table, Journal of the Japan Statistical Society. 14 (1984) 35-42.

[17] S. Tomizawa, A generalization of the marginal homogeneity model for square contingency tables with ordered categories, Journal of Educational and Behavioral Statistics. 20 (1995) 349-360.

[18] S. Tomizawa, N. Miyamoto and M. Ouchi, Decompositions of symmetry model into marginal homogeneity and distance subsymmetry in square contingency tables with ordered categories, Revstat: Statistical Journal. 4 (2006) 153-161. 